\title{
HOMOTOPY GROUPS IN LIE FOLIATIONS
}

\author{
ENRIQUE MACIAS-VIRGÓS
}

\begin{abstract}
According to the results of Fédida and Molino [9], the structure of a $G$-Lie foliation $F$ on a compact manifold $M$ can be described by means of four locally trivial fibre bundles. In this paper we study the relations that those fibrations imply among the (rational) homotopy groups of: the manifold $M$, the generic leaf $L$, its closure $N=\bar{L}$, the basic manifold $W$, the Lie group $G$, and the structural Lie group $H$. Also, we prove that those relations are a particular case of an algebraic result concerning generalized homology theories.
\end{abstract}

\section{INTRODUCTION}

Lie foliations are a fruitful generalization of those defined by a closed 1form, as they play a central role in the theory of Riemannian foliations [9]. The structure of Lie foliations on complete manifolds is nowadays well known (cf. $\S 2$ ). However, a rather difficult problem is to find obstructions to the existence of Lie foliations with given structural data (see [5]), or to classify them for a fixed (co)dimension. Also, it is an open question when foliations with special topological properties (e.g. all leaves are dense and contractible) do exist for a given transverse structure (see [6] and [8]).

Many of the known results on those and related topics involve homotopical and homological properties, and make a wide use of the various fibrations that describe the structure of a Lie foliation.

In this article we systematically study the relations that those fibrations imply among the (rational) homotopy groups of several manifolds and Lie groups. Namely, if $F$ is a $G$-Lie foliation on $M$ with generic leaf $L$, we consider (cf. §) the homotopy exact sequences of:

- the developing map of $F$;

- the fibration defined by the closures $N=\bar{L}$ of the leaves;

- the basic manifold $W$ as an homogeneous space of $G$;

- the developing map of the induced $H$-Lie foliation on $N$.

In $\S 4$ we show how the fundamental groups and holonomy morphisms associated to $F$ are related. In $\S 5$ we focus our attention on higher homotopy groups, and we construct two complexes of abelian groups:

$$
\ldots \rightarrow \pi_{4}(L) \rightarrow \pi_{4}(M) \rightarrow \pi_{4}(W) \rightarrow \pi_{3}(H) \rightarrow \pi_{2}(L) \rightarrow \pi_{2}(M) \rightarrow 0
$$

Received by the editors July 29, 1993.

1991 Mathematics Subject Classification. Primary 57R30; Secondary 55Nxx, 55Q05.

Key words and phrases. Lie foliations, homotopy groups, homology theories. 
and

$$
\ldots \rightarrow \pi_{5}(W) \rightarrow \pi_{4}(H) \rightarrow \pi_{3}(L) \rightarrow \pi_{3}(M) \rightarrow \pi_{3}(W) \rightarrow 0 \rightarrow 0
$$

which we prove (Theorem 5.1) to have isomorphic homology groups.

As a matter of fact, the complexes above are the top and the bottom lines of the following commutative diagram

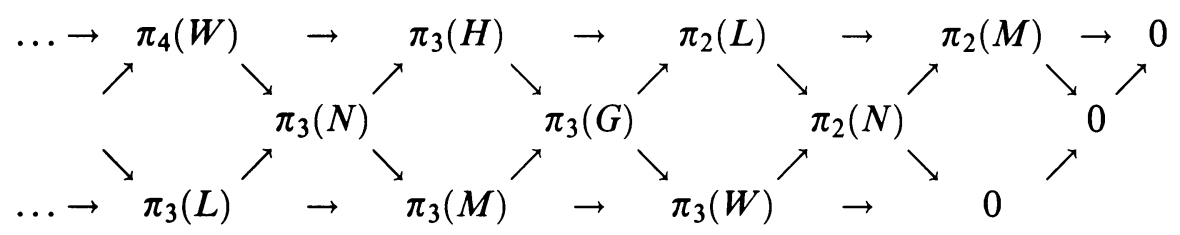

which we call the braid of the Lie foliation $F$; it is built-up from the four homotopy exact sequences defined in $\S 3$. Let us remark that each exact sequence has a sinusoidal shape. Another braid appears when we consider rational homotopy groups $\pi_{n} \otimes \mathbb{Q}$ instead of the abelian groups $\pi_{n}, n \geq 2$.

Then we state several corollaries, to exhibit some nontrivial relations that Theorem 5.1 implies. For example (Proposition 5.2), $M, N$ and $L$ must have isomorphic rational homotopy groups $\pi_{2 n} \otimes \mathbb{Q}$ in even degrees.

Finally, we remark that diagram (1) is a particular case of a more general result (§6) concerning any generalized homology theory [2]. In this paper we do not intend to apply that result to other settings than Lie foliations, but rather to present it as a tool in the study of homotopy groups.

More precisely, let $T$ be a functor, from a suitable category of topological pairs into the category of graded abelian groups, provided with a natural transformation $\partial_{(X, A)}: T(X, A) \rightarrow T(A, \varnothing)$ of degree -1 , such that the following sequence is exact

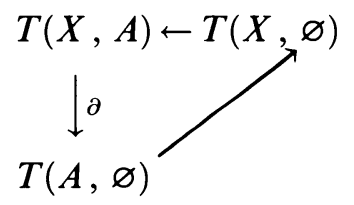

Then, to each triad $B \subset A \subset X$ we associate $t w o$ complexes (depending on whether we take even or odd degrees at $(X, A))$ of abelian groups

$$
\ldots \rightarrow T_{n}(X, A) \stackrel{\partial^{\prime}}{\rightarrow} T_{n-1}(A, B) \stackrel{\partial_{(A, B)}}{\rightarrow} T_{n-2}(B) \stackrel{j_{*}^{\prime}}{\rightarrow} T_{n-2}(X) \stackrel{p_{*}}{\rightarrow} T_{n-2}(X, A) \rightarrow \ldots
$$

which arise from the following commutative diagram of pairs

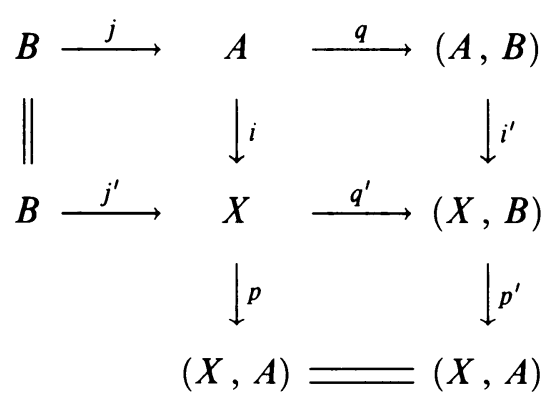

and we prove in $\S 7$ that both complexes have isomorphic homology groups (for suitably taken degrees). 
Acknowledgments. The author thanks Professors D. Tanré and J. C. Thomas for several useful remarks, and all members of the Laboratoire Géométrie-AnalyseTopologie de l'Université de Lille for their hospitality. Professor J. R. Munkres pointed out to me reference [12]. Partial financial support was provided by DGICYT-Spain.

\section{LIE FOLIATIONS}

The structure of Lie foliations on complete manifolds was studied by Hermann [7], Fédida [3] and Molino [9] among others. We now summarize their results and establish our notation.

Let $M$ be a connected complete manifold. If $\omega_{x}: T_{x} M \rightarrow \mathfrak{g}$ is a 1 -form on $M$ with values in the Lie algebra $\mathfrak{g}$, such that

- $\omega$ is of maximal rank $n=\operatorname{dim} \mathfrak{g}$,

- $d \omega+\frac{1}{2}[\omega, \omega]=0$,

then $\operatorname{Ker} \omega_{x}$ is a completely integrable distribution on $M$, and the associated foliation $F$ is called a $\mathfrak{g}$-Lie foliation.

(a) Developing map. There exists a regular covering $p: \widetilde{M} \rightarrow M$ such that the lifting $p^{*} F$ of $F$ to $\widetilde{M}$ is the simple foliation defined by a locally trivial fibre bundle $D: \widetilde{M} \rightarrow G$, for $G$ the simply-connected Lie group associated with $\mathfrak{g}$.

(b) Holonomy morphism. Let $x_{0}, \tilde{x}_{0}$ be fixed base points in $M, \widetilde{M}$, resp., with $p\left(\tilde{x}_{0}\right)=x_{0}, D\left(\tilde{x}_{0}\right)=e \quad(=$ the unit element of $G)$. The representation

$$
h: \pi_{1}\left(M, x_{0}\right) \rightarrow G
$$

given by $h[\alpha]=D(\tilde{\alpha}(1)), \tilde{\alpha}(0)=\tilde{x}_{0}, p \tilde{\alpha}=\alpha$, verifies that $\operatorname{Ker} h=\pi_{1}(\widetilde{M})$.

Moreover, the developing map $D$ is $h$-equivariant, that is,

$$
D(\gamma(x))=D(x) \gamma \quad \forall x \in \widetilde{M}, \gamma \in \Gamma,
$$

where the so-called holonomy group $\Gamma=\operatorname{im} h$ is the group of deck transformations of the covering $p$, acting on $G$ by right translations.

The leaf space of $F$ is then $G / \Gamma$ (a possibly non-Hausdorff space), and there is a commutative diagram

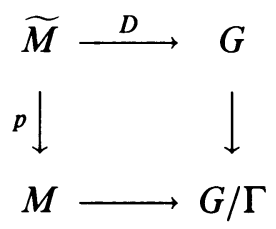

We remark that all leaves of $F$ are diffeomorphic to the fibre $L$ of $D$, and that they are dense in $M$ if and only if $\Gamma$ is dense in $G$.

(c) Basic fibration. Let $\bar{\Gamma}$ be the closure of $\Gamma=\operatorname{im} h$ in $G, \bar{\Gamma}_{e}$ the path connected component of $e$. As for all transversely complete foliations [9], the closures $N=\bar{L}$ of the leaves of $F$ are the fibres of some locally trivial fibre bundle $B: M \rightarrow W$. In our case, $W=G / \bar{\Gamma}$ and $B(x)=[D(\tilde{x})], p(\tilde{x})=x$.

(d) Structural Lie algebra. Let us consider the foliation $F_{0}$ determined by $F$ on each fibre $N=\bar{L}$ of $B$. Then $F_{0}$ turns out to be a h-Lie foliation with dense leaves, for $\mathfrak{h}$ the Lie algebra of the connected Lie group $\bar{\Gamma}_{e}$. 
Let $H$ be the simply-connected Lie group associated with $\mathfrak{h}$. For the induced foliation $F_{0}$ we shall also have a covering $p_{0}: \widetilde{N} \rightarrow N$, a developing map $D_{0}: \widetilde{N} \rightarrow H$, and a holonomy morphism $h_{0}: \pi_{1}(N) \rightarrow H$, with $\Gamma_{0}=\operatorname{im} h_{0}$ the group of deck transformations of $p_{0}$. It is a dense subgroup of $H$ because the leaves of $F_{0}$ are dense in $N$.

Remark 2.1. It is worthy of note that the Lie algebra $\mathfrak{g}$ is not intrinsic, but $h$ certainly is. Suppose the foliation $F$ is defined by another $\mathfrak{g}^{\prime}$-valued form (actually, there are examples with nonisomorphic Lie algebras $\mathfrak{g}^{\prime}$ and $\mathfrak{g}$, see [9]); since the covering $\widetilde{M}$ depends only on $\pi_{1}(L)$, the new developing map would be $D^{\prime}: \widetilde{M} \rightarrow G^{\prime}$, and there is a diffeomorphism $d: G \approx G^{\prime}$ given by $d(g)=D^{\prime}(x)$ with $D(x)=g$. It turns out to be that $d$ is a group isomorphism when restricted to $\Gamma$, so $d$ is a Lie group isomorphism when the leaves are dense. In any case, the homotopy groups $\pi_{n}(G)$ will be structural data.

\section{HOMOTOPY SEQUENCES}

Throughout this section, all group sequences will be exact. They will arise as the homotopy sequences of the several fibrations we associated in $\S 2$ to the Lie foliation $F$.

(a) First, since $\widetilde{M}$ is a covering of $M$, we have $\pi_{n}(M)=\pi_{n}(\widetilde{M})$ for all $n \geq 2$; also, $\pi_{1}(L)=\pi_{1}(\widetilde{M})=\operatorname{Ker} h$ because $\pi_{2}(G)=0$ for all Lie groups. Then we obtain

$$
1 \rightarrow \pi_{1}(L) \rightarrow \pi_{1}(M) \rightarrow \Gamma \rightarrow 1
$$

from the holonomy morphism $h$, and

$$
\ldots \rightarrow \pi_{3}(M) \rightarrow \pi_{3}(G) \rightarrow \pi_{2}(L) \rightarrow \pi_{2}(M) \rightarrow 0
$$

from the developing map $D$.

The same holds for the foliation $F_{0}$, so we have

$$
1 \rightarrow \pi_{1}(L) \rightarrow \pi_{1}(N) \rightarrow \Gamma_{0} \rightarrow 1
$$

and

$$
\ldots \rightarrow \pi_{3}(N) \rightarrow \pi_{3}(H) \rightarrow \pi_{2}(L) \rightarrow \pi_{2}(N) \rightarrow 0 .
$$

(b) Now, there is a long exact sequence

$$
\ldots \rightarrow \pi_{4}(W) \rightarrow \pi_{3}(H) \rightarrow \pi_{3}(G) \rightarrow \pi_{3}(W) \rightarrow 0 .
$$

In fact, since $H$ is the universal covering of $\bar{\Gamma}_{e}$, we have $H / K=\bar{\Gamma}_{e}$ for the central discrete subgroup $K=\pi_{1}\left(\bar{\Gamma}_{e}\right)$ of $H$. Also, $\pi_{n}(H)=\pi_{n}\left(\bar{\Gamma}_{e}\right)$ for all $n \geq 2$. The universal covering of the basic manifold $W=G / \bar{\Gamma}$ is the simplyconnected group $G / \bar{\Gamma}_{e}$, then $\pi_{1}(W)=\bar{\Gamma} / \bar{\Gamma}_{e}$ and $\pi_{n}(W)=\pi_{n}\left(G / \bar{\Gamma}_{e}\right)$ for all $n \geq 2$. But $\pi_{2}=0$ for the Lie group $\bar{\Gamma}_{e}$, so sequence (7) arises from the fibration $G \rightarrow G / \bar{\Gamma}_{e}$, because $\pi_{2}(W)=\pi_{1}\left(\bar{\Gamma}_{e}\right)=K$.

(c) Finally, we study the homotopy sequence of the basic fibration $B$. We will have

$$
0 \rightarrow \pi_{2}(W) \rightarrow \pi_{1}(N) \rightarrow \pi_{1}(M) \rightarrow \pi_{1}(W) \rightarrow 0
$$

and

$$
\ldots \rightarrow \pi_{3}(M) \rightarrow \pi_{3}(W) \rightarrow \pi_{2}(N) \rightarrow \pi_{2}(M) \rightarrow 0 .
$$


To prove this, we remark that the morphism $B_{\#}: \pi_{2}(M) \rightarrow \pi_{2}(W)$ factors through $\pi_{2}(G)=0$ via the developing map $D$, because $p_{\#}: \pi_{2}(\widetilde{M}) \cong \pi_{2}(M)$ is an isomorphism for the covering $p$, and $B(x)=[D(\tilde{x})], p(\tilde{x})=x$. So $B_{\#}=0$ at degree 2 , which means that the connecting morphism $\partial: \pi_{2}(W) \rightarrow \pi_{1}(N)$ is injective. In other words, some intermediate covering of $M$ fibres over $G / \bar{\Gamma}_{e}$.

\section{Fundamental GROUPS}

The results in this section will not be used elsewhere, but they clarify the relations among the various groups we have introduced. They explain why the abelian groups $\pi_{n}, n \geq 2$ (excepting $\pi_{2}(W)$ ) serve to build-up a complex (cf. §5) which has nothing to do with fundamental groups. On the other hand, $\pi_{2}(W)$ can be identified with a central subgroup $K$ of $H$.

As we have seen in $\S 3$, the connecting morphism $\partial: \pi_{2}(W) \rightarrow \pi_{1}(N)$ in the homotopy sequence of the basic fibration is injective. Then we have an exact sequence

$$
0 \rightarrow \pi_{2}(W) \rightarrow \pi_{1}(N) \stackrel{i_{\#}}{\rightarrow} \pi_{1}(M) \stackrel{B_{\sharp}}{\rightarrow} \pi_{1}(W) \rightarrow 1
$$

with $\pi_{1}(W)=\bar{\Gamma} / \bar{\Gamma}_{e}, \pi_{2}(W)=\pi_{1}\left(\bar{\Gamma}_{e}\right)$.

Proposition 4.1. (a) The image of $\pi_{1}(N) \stackrel{i_{\sharp}}{\rightarrow} \pi_{1}(M) \stackrel{h}{\rightarrow} \Gamma$ is $\Gamma \cap \bar{\Gamma}_{e}$.

(b) The morphism $\pi_{2}(W) \stackrel{\partial}{\rightarrow} \pi_{1}(N) \stackrel{h_{0}}{\rightarrow} \Gamma_{0}$ is the inclusion $K=\pi_{1}\left(\bar{\Gamma}_{e}\right) \subset \Gamma_{0}$.

Moreover, for the developing map $D: \widetilde{M} \rightarrow G$, the inverse image $D^{-1}\left(\bar{\Gamma}_{e}\right)$ is a covering of $N=\bar{L}$, with group $\Gamma \cap \bar{\Gamma}_{e}$ of deck transformations, and $\Gamma_{0} / K=\Gamma \cap \bar{\Gamma}_{e}$ while $H / K=\bar{\Gamma}_{e}$.

Then we have the following commutative diagram of groups with exact rows and columns

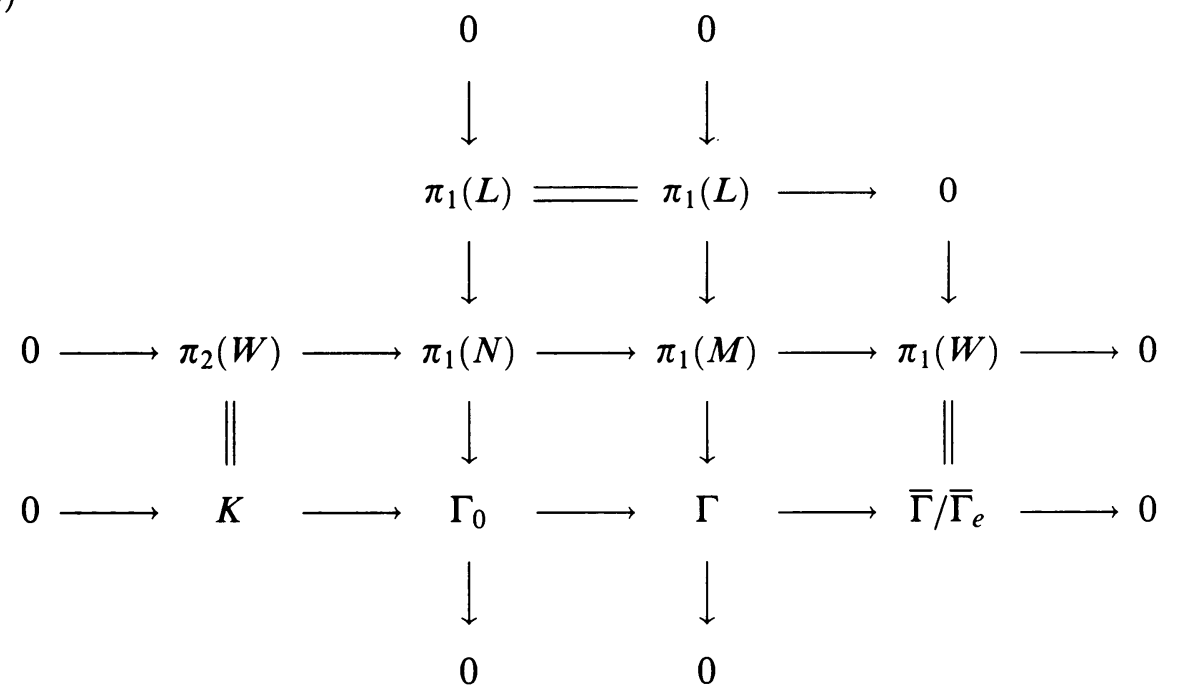

Corollary 4.2. (a) There is an exact sequence of groups $1 \rightarrow \Gamma \cap \bar{\Gamma}_{e} \rightarrow \Gamma \rightarrow$ $\bar{\Gamma} / \bar{\Gamma}_{e} \rightarrow 1$, that is, $\forall \bar{\gamma} \in \bar{\Gamma}, \exists \gamma \in \Gamma$ such that $\bar{\gamma}=\gamma \cdot \alpha(1)$ for some path $\alpha$ in $\bar{\Gamma}$ with $\alpha(0)=e$.

(b) $\bar{\Gamma}=\Gamma \cdot \bar{\Gamma}_{e}$. 
(c) $\bar{\Gamma}_{e} /\left(\Gamma \cap \bar{\Gamma}_{e}\right)=\bar{\Gamma} / \Gamma$.

(d) $\Gamma \cap \bar{\Gamma}_{e}$ is dense in $\bar{\Gamma}_{e}$.

\section{THE BRAID OF A LiE FOliation}

We now merge the four exact sequences (4), (6), (7) and (9) of $\S 3$, to obtain the braid of the Lie foliation $F$ (diagram (1) in $\S 1$ ).

It turns out that diagram (1) is commutative, because we have identified the higher homotopy groups of each manifold with those of some covering.

So, to each Lie foliation we can attach $t w o$ complexes (depending on whether we take even or odd degrees at $W$ ) of abelian groups:

$$
\ldots \rightarrow \pi_{n}(L) \rightarrow \pi_{n}(M) \rightarrow \pi_{n}(W) \rightarrow \pi_{n-1}(H) \rightarrow \pi_{n-2}(L) \rightarrow \ldots
$$

ending at $\ldots \rightarrow \pi_{2}(M) \rightarrow 0$ ( $n$ even) or $\ldots \rightarrow \pi_{3}(W) \rightarrow 0$ ( $n$ odd), which correspond to the bottom and top lines of diagram (1).

Theorem 5.1. (a) The two complexes (11) have isomorphic homology groups.

(b) The same result is true if we consider rational homotopy groups $\pi_{n}(X) \otimes \mathbb{Q}$ and complexes of rational vector spaces (instead of the abelian groups $\pi_{n}(X)$, $n \geq 2$ ).

For a fibration $F \rightarrow E \rightarrow B$ we identify $\pi_{n}(E, F)$ with $\pi_{n}(B)$. In this manner Theorem 5.1 becomes a particular case of a more general result concerning generalized homology theories, that we will prove in $\$ 7$. In order to understand the analogy with diagram (2) in $\S 1$, one can imagine that there is an (up to homotopy) commutative diagram

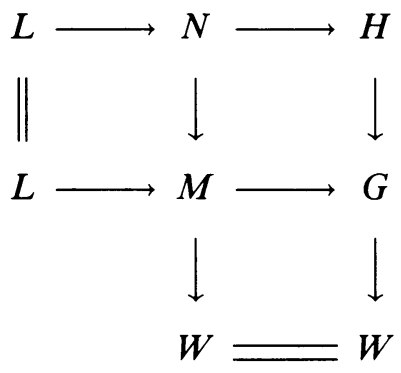

We now prove some consequences of Theorem 5.1.

Proposition 5.2. For any Lie foliation we have:

(a) The inclusions induce isomorphisms $\pi_{2 n}(L) \otimes \mathbb{Q} \cong \pi_{2 n}(\bar{L}) \otimes \mathbb{Q} \cong \pi_{2 n}(M) \otimes \mathbb{Q}$ for all $n \geq 0$;

(b) The projection induces epimorphisms $\pi_{2 n+1}(M) \otimes \mathbb{Q} \rightarrow \pi_{2 n+1}(W) \otimes \mathbb{Q}$ for all $n \geq 1$

(c) For each $n \geq 2$ there is the following commutative diagram, with exact 
rows and columns:

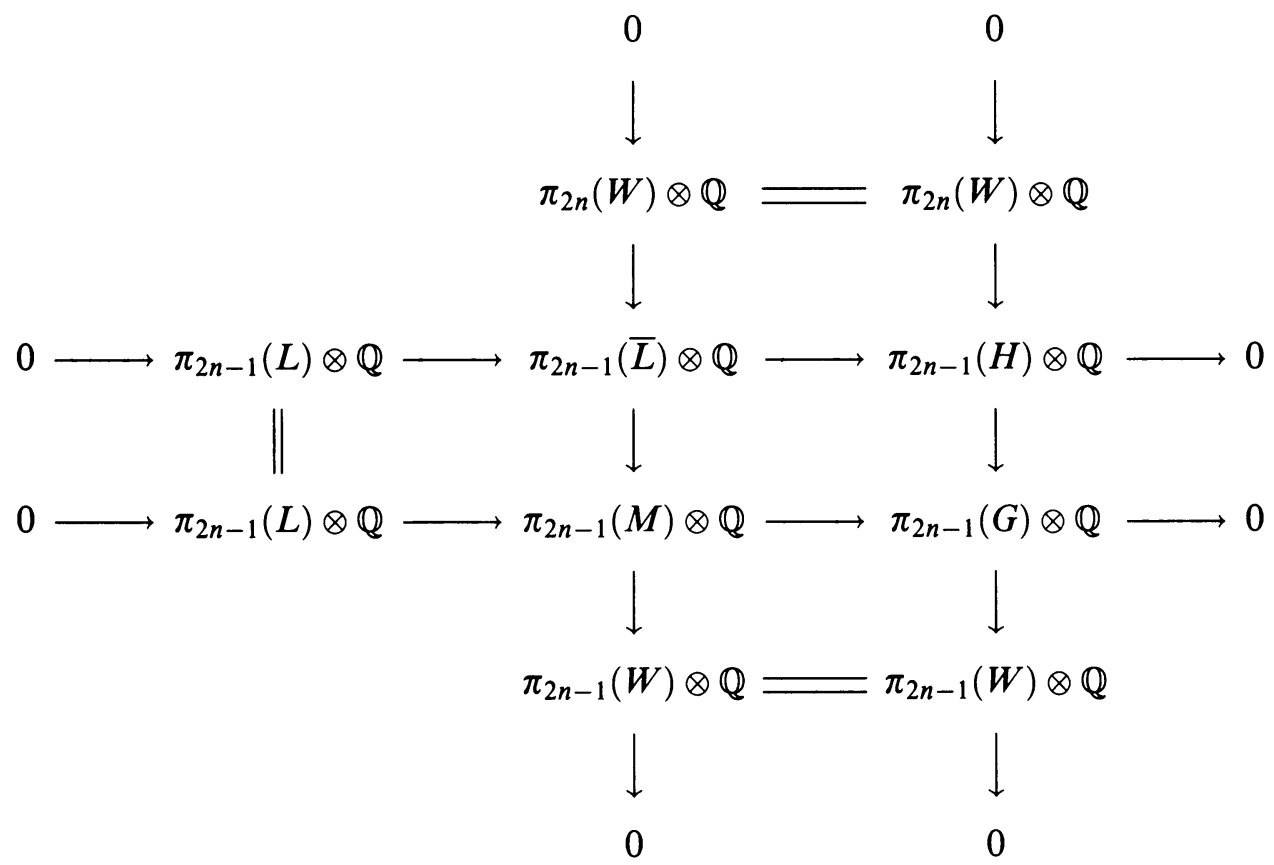

Proof. For the Lie groups $G$ and $H, \pi_{n} \otimes \mathbb{Q}=0 \forall n \geq 2$. Moreover [4], the rational connecting morphism is zero in odd degrees for the fibrations with simply-connected base $D: \widetilde{M} \rightarrow G$ and $D_{0}: \widetilde{N} \rightarrow H$.

Corollary 5.3. For the rational homotopy groups of a Lie flow, we have

(a) $\pi_{2 n}(M) \otimes \mathbb{Q}=0 \quad \forall n \geq 1$;

(b) $\pi_{2 n}(W) \otimes \mathbb{Q}=0 \quad \forall n \geq 2$;

(c) $\pi_{2 n+1}(M) \otimes \mathbb{Q} \cong \pi_{2 n+1}(G) \otimes \mathbb{Q} \cong \pi_{2 n+1}(W) \otimes \mathbb{Q} \quad \forall n \geq 1$.

Proof. For a Lie flow (that is $L=\mathbb{R}$ or $L=S^{1}$ ), the closure $N$ of any leaf is a torus [1].

Proposition 5.4. For a G-Lie foliation, the following statements are equivalent:

(a) $G$ is a contractible Lie group;

(b) $\pi_{n}(L) \cong \pi_{n}(M) \forall n \geq 2$;

(c) The inclusion $L \subset M$ induces an isomorphism $\pi_{2}(L) \cong \pi_{2}(M)$ and an epimorphism

$$
\pi_{3}(L) \rightarrow \pi_{3}(M)
$$

(d) The inclusion $N \subset M$ induces an isomorphism $\pi_{2}(N) \cong \pi_{2}(M)$ and the following are epimorphisms:

$$
\pi_{3}(\bar{L}) \rightarrow \pi_{3}(M), \quad \pi_{4}(G / \bar{\Gamma}) \rightarrow \pi_{3}(\bar{\Gamma}) .
$$

Proof. It is well known that a Lie group $G$ is contractible if and only if $\pi_{1}(G)$ and $\pi_{3}(G)$ are trivial.

We remark that the maps (13) and (14) are always injective in rational homotopy because $\pi_{4}(G) \otimes \mathbb{Q}=0$. There are analogous conditions for the structural Lie group $H$ to be contractible. 
Also remark that for a nilpotent foliation on a compact manifold, $\bar{\Gamma}_{e}$ is a normal subgroup of $G$, then $\pi_{2}(W)=0$.

\section{HOMOLOGY THEORIES}

In order to prove Theorem 5.1 it is easiest to work in the more general setting of homology theories. Usually, a homology theory is defined as a functor $T$, from a suitable category of topological pairs into the category of graded abelian groups, which is subject to several axioms [2]. We will consider a generalized version of those homology theories, as we only impose the following

Axiom 6.1. For each $n \in Z$ there exists a natural transformation

$$
\partial_{(X, A)}: T_{n}(X, A) \rightarrow T_{n-1}(A)
$$

such that the following sequence is exact,

$$
\ldots \rightarrow T_{n}(A) \stackrel{i_{*}}{\rightarrow} T_{n}(X) \stackrel{p_{*}}{\rightarrow} T_{n}(X, A) \stackrel{\partial}{\rightarrow} T_{n-1}(A) \rightarrow \ldots
$$

for $i:(A, \varnothing) \rightarrow(X, \varnothing)$ and $p:(X, \varnothing) \rightarrow(X, A)$.

Well-known examples of such homology theories are singular homology (with abelian coefficients), homotopy and rational homotopy groups. Sequence (15) is called the long exact sequence of the pair $(X, A)$. As it is customary, we denote $\phi_{*}$ the group morphism $T_{n}(\phi): T_{n}(X, A) \rightarrow T_{n}(Y, B)$ corresponding to a map $\phi:(X, A) \rightarrow(Y, B)$, and we write $T_{n}(X)$ instead of $T_{n}(X, \varnothing)$. A direct consequence of the axiom above is that $T_{n}(X, X)=0$ for all $X$.

To each triad $B \subset A \subset X$ we associate the following commutative diagram

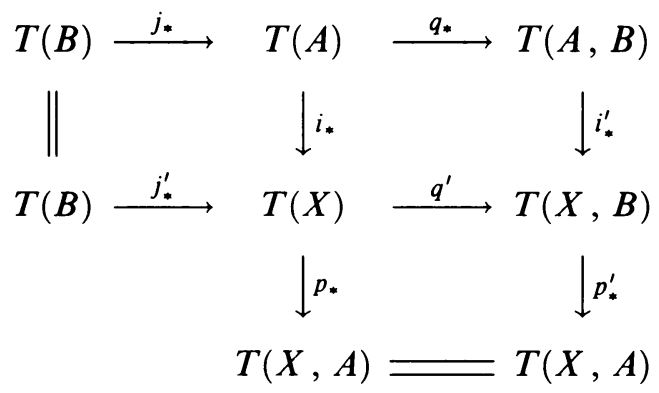

which involves four long exact sequences. Three of them correspond to the pairs $(A, B),(X, A)$ and $(X, B)$. The fourth is the so-called long sequence of the triad $B \subset A \subset X$,

$$
\ldots \rightarrow T_{n}(A, B) \stackrel{i_{*}^{\prime}}{\rightarrow} T_{n}(X, B) \stackrel{p_{*}^{\prime}}{\rightarrow} T_{n}(X, A) \stackrel{\partial^{\prime}}{\rightarrow} T_{n-1}(A, B) \rightarrow \ldots
$$

where $\partial^{\prime}$ is the composition

$$
T_{n}(X, A) \stackrel{\partial_{(X, A)}}{\rightarrow} T_{n-1}(A) \stackrel{q_{*}}{\rightarrow} T_{n-1}(A, B) .
$$

By only using the axiom above, it is not hard to prove that the long sequence of any triad $B \subset A \subset X$ is exact [2]. Of course, when $B, A$ or $X$ turn out to be $\varnothing$ one recovers the exact sequence of a pair.

\section{BRAIDS}

We shall associate to each triad $B \subset A \subset X$ two complexes of abelian groups which will have isomorphic homology groups. 
For each $n \in \mathbb{Z}$ let us consider the sequence

$$
\ldots \rightarrow T_{n}(X, A) \stackrel{\partial^{\prime}}{\rightarrow} T_{n-1}(A, B) \stackrel{\partial_{(A, B)}}{\rightarrow} T_{n-2}(B) \stackrel{j_{*}^{\prime}}{\rightarrow} T_{n-2}(X) \stackrel{p_{*}}{\rightarrow} T_{n-2}(X, A) \rightarrow \ldots
$$

which arises from diagram (16). Actually, we obtain two sequences

$$
\ldots \rightarrow T_{2}(X, A) \rightarrow T_{1}(A, B) \rightarrow T_{0}(B) \rightarrow T_{0}(X) \rightarrow T_{0}(X, A) \rightarrow \ldots
$$

and

$$
\ldots \rightarrow T_{1}(B) \rightarrow T_{1}(X) \rightarrow T_{1}(X, A) \rightarrow T_{0}(A, B) \rightarrow T_{-1}(B) \rightarrow \ldots
$$

depending on whether we take even or odd degrees at $(X, A)$.

Our main result in this section is that the complexes (17) and (18) have the same homology groups, at corresponding places when the degrees are suitably chosen. To avoid tedious notations, we will denote $\mathrm{H} T_{n}(Y, Z)$ the homology group of each complex (17) or (18) at the place $T_{n}(Y, Z)$, for $(Y, Z)$ a topological pair. For instance, $\mathrm{H} T_{0}(B)=\operatorname{Ker} f / \operatorname{Im} g$ for $T_{1}(A, B) \stackrel{f}{\rightarrow} T_{0}(B) \stackrel{g}{\rightarrow}$ $T_{0}(X)$ in complex (17).

Theorem 7.1. (a) $\mathrm{H} T_{n+1}(X, A)=\mathrm{H} T_{n}(B)$.

(b) $\mathrm{H} T_{n}(X)=\mathrm{H} T_{n}(A, B)$.

In order to prove Theorem 7.1, we begin by merging the four exact sequences associated to the triad $B \subset A \subset X$. We obtain the following diagram, which generalizes diagram (1). We remark that each exact sequence has a sinusoidal shape, while the top and bottom lines are just the sequences (17) and (18):

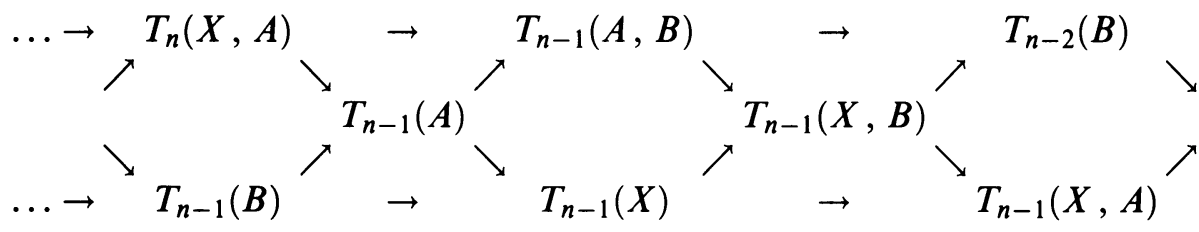

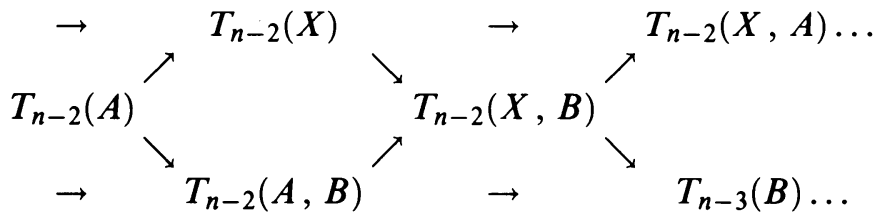

Remark 7.2. One could consider a third complex

$$
\ldots \rightarrow T_{n-1}(A) \rightarrow T_{n-1}(X, B) \rightarrow T_{n-2}(A) \rightarrow \ldots
$$

for which there are vertical chain maps to complexes (17), (18), that induce the null morphism in homology.

Proposition 7.3. Diagram (19) is commutative, and the sequences (17) and (18) are complexes of abelian groups.

The proof is straightforward.

Remark 7.4. According to Munkres [11, Ex. 1, p. 148], we call diagram (19) a braid. To the best of our knowledge, it appeared for the first time in [12], where 
it was due to Kervaire. In Wall's article, the diagram was a tool for studying the exactness of the four involved sequences. An analogous diagram appears in the setting of $C W$-complexes as a part of the spectral sequence associated to the filtration by the skeletons. See for example [10].

Proof of Theorem 7.1. We will give a general proof of Theorem 7.1 for any commutative diagram

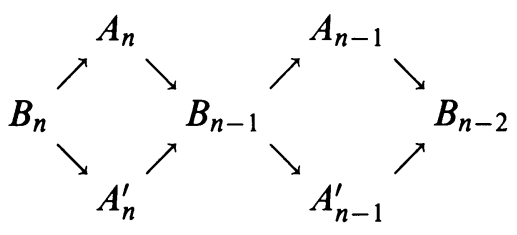

where all sequences

$$
\ldots \rightarrow A_{n+2}^{\prime} \stackrel{\alpha_{n+2}^{\prime}}{\rightarrow} B_{n+1} \stackrel{\beta_{n+1}}{\rightarrow} A_{n+1} \stackrel{\beta_{n} \alpha_{n+1}}{\rightarrow} A_{n} \stackrel{\alpha_{n}}{\rightarrow} B_{n-1} \stackrel{\beta_{n-1}^{\prime}}{\rightarrow} A_{n-1}^{\prime} \stackrel{\beta_{n-2} \alpha_{n-1}}{\rightarrow} \ldots
$$

are supposed to be exact.

Let $A$ be the complex $A_{n}$, with differential $\partial_{n}^{A}: A_{n} \rightarrow A_{n-1}$ given by $\partial_{n}=\beta_{n-1} \alpha_{n}$. Analogously, we define $B=\left(B_{n}, \partial^{B}\right)$ with $\partial_{n}^{B}=\alpha_{n} \beta_{n}=\alpha_{n}^{\prime} \beta_{n}^{\prime}$, and $A^{\prime}=\left(A_{n}^{\prime}, \partial^{A^{\prime}}\right)$ with $\partial_{n}^{\prime}=\beta_{n-1}^{\prime} \alpha_{n}^{\prime}$.

One can check in all cases that $\partial_{n-1} \partial_{n}=0$.

We are interested in the homology groups of the three complexes $A, B$ and $A^{\prime}$. It is rather obvious that $\alpha, \alpha^{\prime}, \beta$, and $\beta^{\prime}$ are chain maps, so we have morphisms

$$
\begin{aligned}
& \alpha_{*}: H_{n}(A) \rightarrow H_{n-1}(B), \quad \alpha_{*}^{\prime}: H_{n}\left(A^{\prime}\right) \rightarrow H_{n-1}(B), \\
& \beta_{*}: H_{n}(B) \rightarrow H_{n}(A), \quad \beta_{n}^{\prime}: H_{n}(B) \rightarrow H_{n}\left(A^{\prime}\right),
\end{aligned}
$$

such that

$$
\beta_{* n-1} \alpha_{* n}=\beta_{* n-1}^{\prime} \alpha_{* n}=\alpha_{* n-1}^{\prime} \beta_{* n}=\beta_{* n-1}^{\prime} \alpha_{* n}^{\prime}=0 .
$$

Proposition 7.5. There exist unique inverse isomorphisms $f_{*}: H(A) \cong H\left(A^{\prime}\right)$ and $g_{*}: H\left(A^{\prime}\right) \cong H(A)$ such that $\alpha_{*}^{\prime} f_{*}=\alpha_{*}, f_{*} b_{*}=\beta_{*}^{\prime}$ and $g_{*} \beta_{*}^{\prime}=\beta_{*}$, $\alpha_{*} g_{*}=\alpha_{*}^{\prime}$.

Proof. Indeed, this is equivalent to the butterfly lemma in [10, p. 71] or braid lemma in [11, p. 148].

Let $[x]$ be in $H_{n}(A)$, that is $x \in A_{n}$ and $\partial^{A}(x)=0$. Then $\beta_{n-1} \alpha_{n}(x)=$ 0 . By exactness at $B_{n-1}, \exists z \in A_{n}^{\prime}$ such that $\alpha_{n}^{\prime}(z)=\alpha_{n}(x)$. Let us define $f_{*}[x]=[z] \in H_{n}\left(A^{\prime}\right)$, with $z$ being a cycle because

$$
\partial^{A^{\prime}}(z)=\beta_{n-1}^{\prime} \alpha_{n}^{\prime}(z)=\beta_{n-1}^{\prime} \alpha_{n}(x)=0 .
$$

To see that $f_{*}$ is well defined, let us take $z^{\prime} \in A_{n}^{\prime}$ with $\alpha_{n}^{\prime}\left(z^{\prime}\right)=\alpha_{n}(x)$. Then $\alpha_{n}^{\prime}\left(z^{\prime}-z\right)=0$ so that $z^{\prime}-z=\partial_{n+1}(w), w \in A_{n+1}^{\prime}$, and hence $\left[z^{\prime}\right]=[z]$.

Moreover, if $x=\partial^{A}(y), y \in A_{n+1}$ then $x=\beta_{n} \alpha_{n+1}(y), \alpha_{n+1}(y) \in B_{n}$, so $f_{*}[x]=[z]$ for $z=\beta_{n}^{\prime} \alpha_{n+1}^{\prime}(y)$ because

$$
\alpha_{n}^{\prime} \beta_{n}^{\prime} \alpha_{n}(y)=\alpha_{n} \beta_{n} \alpha_{n+1}(y)=\alpha_{n}(x) ;
$$

but $z=\beta_{n}^{\prime} \alpha_{n+1}(y)=0$ by exactness at $B_{n}$.

The definition obviously implies that $\alpha_{n *}^{\prime} f_{*}=\alpha_{n *}$. 
Also, $\partial(x)=0$ implies that $\exists a \in B_{n}$ with $\beta_{n}(a)=x$ by exactness at $A_{n}$, and

$$
\partial(a)=\alpha_{n} \beta_{n}(a)=\alpha_{n}^{\prime} \beta_{n}^{\prime}(a)
$$

thus, $f_{*}[x]=\left[\beta_{n}^{\prime}(a)\right]$. This shows $f_{*} \beta_{*}=\beta_{*}^{\prime}$.

Now we observe that for any other $a^{\prime} \in B_{n}$ with $\beta_{n}\left(a^{\prime}\right)=x$, we have $f_{*}[x]=\left[\beta_{n}\left(a^{\prime}\right)\right]$ because $\beta_{n}\left(a^{\prime}-a\right)=0$, that is $a^{\prime}-a=\alpha_{n+1}^{\prime}(w), w \in A_{n+1}^{\prime}$ by exactness at $B_{n}$. Then

$$
\beta_{n}^{\prime}\left(a^{\prime}-a\right)=\beta_{n}^{\prime} \alpha_{n+1}^{\prime}(w)=\partial^{A^{\prime}}(w)
$$

This gives an alternative definition of $f_{*}$.

In exactly the same way we can define and handle $g_{*}$. To prove $f_{*} g_{*}=\mathrm{id}$, $g_{*} f_{*}=\mathrm{id}$, we use the alternative definitions to see that $f_{*}[x]=[z], \alpha_{n}^{\prime}(z)=$ $\alpha_{n}(x)$, imply $g_{*}[z]=[x]$.

Uniqueness is easy to prove.

\section{REFERENCES}

1. P. Caron and Y. Carrière, Flots transversalement de Lie, C. R. Acad. Sci. Paris 280 (1980), 477-478.

2. S. Eilenberg and N. E. Steenrod, Foundations of algebraic topology, Princeton Univ. Press, 1952.

3. E. Fédida, Sur les feuilletages de Lie, C. R. Acad. Sci. Paris 277 (1971), 999-1002.

4. Y. Félix, La dichotomie elliptique-hyperbolique en homotopie rationnelle, Astérisque 176, S.M.F., 1989.

5. E. Gallego and A. Reventós, Lie flows of codimension 3, Trans. Amer. Math. Soc. 326 (1991), 529-541.

6. E. Ghys, Groupes d'holonomie des feuilletages de Lie, Indag. Math. 47 (1985), 173-182.

7. R. Hermann, On the differential geometry of foliations, Ann. of Math. (2) 72 (1960), 445-457.

8. G. Meigniez, Sous-groupes de génération compacte des groupes de Lie résolubles, Université Paris VII, preprint, 1992.

9. P. Molino, Riemannian foliations, Progress in Math., 73, Birkhäuser, 1988.

10. R. E. Mosher and M. C. Tangora, Cohomology operations and applications in homotopy theory, Harper \& Row, 1968.

11. J. R. Munkres, Elements of algebraic topology, Addison-Wesley, 1984.

12. C. T. C. Wall, On the exactness of interlocking sequences, Enseign. Math. 12 (1966), 95-100.

Departamento de Xeometria e Topoloxia, Facultade de Matemáticas, Universidade de Santiago de Compostela, 15706 Galiza, Spain

E-mail addresses: emaciasv@seins.usc.es

macias@zmat .usc.es 